\title{
FRACTIONAL PART SUMS AND LATTICE POINTS
}

\author{
by WERNER GEORG NOWAK*
}

(Received 23rd September 1996)

The objective of this article are sums $S(M)=\sum_{n} \psi(M f(n / M))$ where $\psi$ denotes essentially the fractional part minus $\frac{1}{2}, f$ is a $C^{4}$-function with $f^{\prime \prime}$ nonvanishing, and summation is extended over an interval of order $M$. For $S(M)$ an $\Omega$-estimate and a mean-square bound is obtained. Applications to problems concerning the number of lattice points in large planar domains are discussed.

1991 Mathematics subject classification: 11P21, $11 \mathrm{~K} 99$.

\section{Introduction and statement of results}

Let $f$ be a real-valued function defined on an interval $[a, b]$ with continuous derivatives up to order 4 and the property that $f^{\prime \prime}$ has no zero on $[a, b]$. Further, let $\psi$ denote a row-of-teeth function satisfying

$$
\begin{aligned}
\psi(t)=t-[t]-\frac{1}{2} & \text { for } t \notin \mathbb{Z}, \\
-\frac{1}{2} \leq \psi(t) \leq \frac{1}{2} & \text { for } t \in \mathbb{Z} .
\end{aligned}
$$

Finally, let $M$ be a large real parameter. Then the objective of the present article will be to study the sum

$$
S(M)=\sum_{a M<n \leq b M, n \in \mathbf{Z}} \psi\left(M f\left(\frac{n}{M}\right)\right) .
$$

It is clear that $S(M)$ is connected with the problem of counting the lattice points in the Euclidean $(\xi, \eta)$-plane between the curve

$$
\frac{\eta}{M}=f\left(\frac{\xi}{M}\right)
$$

and the $\xi$-axis (see Section 2 of this paper). In classic times, upper estimates for sums of this type have been obtained by Vinogradov [26] and van der Corput $[4,5]$, who ultimately proved that

$$
S(M) \ll M^{2 / 3-\varepsilon_{0}}
$$

- This article is part of a research project supported by the Austrian Science Foundation (Nr. P 9892-PHY). 
with some (very small) $\varepsilon_{0}>0$. Under the general conditions stated, there was no improvement until recently when Huxley deduced his "Discrete Hardy Littlewood Method" from earlier ideas due to Bombieri and Iwaniec [1] and Iwaniec and Mozzochi [15]. Huxley's sharpest version [11] contains the bound

$$
S(M) \ll M^{46 / 73}(\log M)^{315 / 146}
$$

(under the additional technical condition that $f^{(3)}$ has no zero on $[a, b]$ ). For a full account, the reader is referred to Huxley's textbook [13]. We remark parenthetically that a more general sum which contains a second parameter $T \geq M$, namely

$$
S(M, T)=\sum_{a M<n \leq b M, n \in \mathbf{Z}} \psi\left(T f\left(\frac{n}{M}\right)\right)
$$

is important for rounding error estimations in connection with classic numerical integration methods. This, too, has been worked out by Huxley [10]. The upper bounds he established depend in a complicated way on the relative size of $T$ and $M$ and are given in a table exceeding one printed page.

The objective of the present article is to complement the known upper estimate for $S(M)$ by a lower bound (a so-called Omega-result) and a mean-square estimate: Roughly speaking, we shall prove that

and

$$
S(M)=\Omega\left(M^{1 / 2}(\log M)^{1 / 4}\right)
$$

$$
\int_{X}^{2 X}(S(M))^{2} \mathrm{~d} M \ll X^{2}
$$

We may thus formulate the interpretation that $S(M) \ll M^{1 / 2}$ "on average", with an unbounded sequence of $M$-values for which $S(M)$ is "exceptionally large".

Before stating our assertions precisely we have to formulate one more condition which will be important in our argument and is most efficiently stated in geometric terms.

Definition. We shall say that a smooth curve $\mathcal{C}$ in the plane satisfies the tangent condition if none of the tangents of $\mathcal{C}$ contains the origin.

Furthermore, we shall establish the $\Omega$-bound for a family of functions $f_{1}, \ldots, f_{J}$ (and corresponding $\psi_{1}, \ldots, \psi_{J}$ ). This will be meaningful for the applications to lattice point problems discussed in Section 2.

Theorem 1. For $j=1, \ldots, J$, let $f_{j}:\left[a_{j}, b_{j}\right] \rightarrow \mathbb{R}$ be functions with four continuous derivatives such that the second derivatives $f_{j}^{\prime \prime}$ have no zero and are all of the same sign. Let $\psi_{j}$ be row-of-teeth functions satisfying (1.1). Suppose that the graphs of $\eta=f_{j}(\xi)$, $\xi \in\left[a_{j}, b_{j}\right]$, all fulfil the tangent condition. Let $M$ be a large real parameter and put 


$$
\mathcal{S}(M)=\sum_{j=1}^{J} \sum_{a_{j} M<n \leq b_{j} M} \psi_{j}\left(M f_{j}\left(\frac{n}{M}\right)\right)
$$

Then it follows that

$$
\lim _{M \rightarrow \infty} \sup \left(\frac{-\operatorname{sgn}\left(f_{j}^{\prime \prime}\right) \mathcal{S}(M)}{M^{1 / 2}(\log M)^{1 / 4}}\right)>0
$$

where $\operatorname{sgn}\left(f_{j}^{\prime \prime}\right)$ denotes the sign of the $f_{j}^{\prime \prime}$.

In fact, we shall obtain a positive lower bound for this limsup which may depend on $J$ and on the ranges of values attained by the functions $f_{j}$ and their first four derivatives. (This applies to all constants, either occurring explicitly or implied by some order-of-magnitude symbol, throughout the paper.)

Theorem 2. With the notation and assumptions of Theorem 1 , it follows that, for $X$ large,

$$
\int_{X}^{2 X}(S(M))^{2} \mathrm{~d} M \ll X^{2},
$$

Remark. The substitution $M \rightarrow M^{0}$, with any fixed $\theta>0$, readily gives

$$
\int_{X}^{2 X}\left(\mathcal{S}\left(M^{0}\right)\right)^{2} \mathrm{~d} M \ll X^{3+0}
$$

Further, by splitting up into dyadic subintervals, the estimate can be extended trivially to $[0, X]$ as range of integration.

\section{Applications to lattice point problems}

In the classic literature the connection between fractional part sums and problems of estimating the number of lattice points in large regions occurs, for one of the first times, in the context of the Gaussian circle problem. (For a thorough account on its history, the reader may consult, e.g., the book of Krätzel [17].) Let $r(n)$ the number of ways to write $n \in \mathbb{N}_{0}$ as a sum of two squares, i.e.,

$$
r(n)=\#\left\{(u, v) \in \mathbb{Z}^{2}: u^{2}+v^{2}=n\right\},
$$

then it is well-known that

$$
\sum_{0 \leq n \leq M^{2}} r(n)=\pi M^{2}+\mathcal{R}(M)
$$


with

$$
\mathcal{R}(M)=-8 \sum_{0<n \leq \frac{M}{\sqrt{2}}} \psi_{*}\left(M \sqrt{1-\left(\frac{n}{M}\right)^{2}}\right)+O(1)
$$

$\psi_{*}(t)=t-[t]-\frac{1}{2}$ throughout. Thus it is immediate from our Theorems that

$$
\mathcal{R}(M)=\Omega_{-}\left(M^{1 / 2}(\log M)^{1 / 4}\right)
$$

and, for any fixed $\theta>0$,

$$
\int_{0}^{x}\left(\mathcal{R}\left(M^{0}\right)\right)^{2} \mathrm{~d} M \ll X^{1+0}
$$

In fact, these two estimates are essentially the best of their kinds known to date: The sharpest lower bound was established by Hafner [7] and reads

$$
\mathcal{R}(M)=\Omega_{-}\left(M^{1 / 2}(\log M)^{1 / 4}(\log \log M)^{\frac{1}{4} \log 2} \exp (-c \sqrt{\log \log \log M})\right) \quad(c>0),
$$

while Kátai [16] obtained the mean-square asymptotic

$$
\int_{0}^{X}\left(\mathcal{R}\left(M^{1 / 2}\right)\right)^{2} \mathrm{~d} M=C X^{3 / 2}+O\left(X(\log X)^{2}\right), \quad C=\frac{1}{3 \pi^{2}} \sum_{n=1}^{\infty} \frac{(r(n))^{2}}{n^{3 / 2}} .
$$

However, our results apply to a much larger class of planar domains $\mathcal{D}$ : Suppose that $\mathcal{C}=\partial \mathcal{D}$ is a closed piecewise smooth curve which can be written in polar coordinates $(r, \lambda)$ as

$$
\mathcal{C}: \quad r=\rho(\lambda), \quad 0 \leq \lambda \leq 2 \pi,
$$

where $\rho$ is continuous on $[0,2 \pi]$ and $\rho(0)=\rho(2 \pi)$. Assume further that $[0,2 \pi]$ can be subdivided by an increasing sequence

$$
0=\lambda_{0}<\lambda_{1}<\ldots<\lambda_{J}=2 \pi
$$

such that each restriction $\rho_{j}$ to an interval $\left[\lambda_{j-1}, \lambda_{j}\right]$ possesses four continuous derivatives on $\left[\lambda_{j-1}, \lambda_{j}\right]$. Suppose finally that each of the curves

$$
\mathcal{C}_{j}: \quad r=\rho_{j}(\lambda), \quad \lambda_{j-1} \leq \lambda \leq \lambda_{j}, \quad j=1, \ldots, J,
$$

has finite nonvanishing curvature throughout and satisfies the tangent condition.

We consider now the planar domain $\mathcal{D}$ which lies "inside" the Jordan curve $\mathcal{C}$ and submit it to a homothetic transformation by a large real factor $M$ : 


$$
M \mathcal{D}=\left\{(u, v) \in \mathbb{R}^{2}: \quad\left(\frac{u}{M}, \frac{v}{M}\right) \in \mathcal{D}\right\}
$$

We denote by $N_{\mathcal{D}}(M)$ the number of lattice points of $\mathbb{Z}^{2}$ which belong to $M \mathcal{D}$, where the lattice points on the boundary $M C$ may get any weight $\omega, 0 \leq \omega \leq 1$. The "lattice rest" or "discrepancy" is then defined as

$$
\mathcal{R}_{\mathcal{D}}(M)=N_{\mathcal{D}}(M)-\operatorname{area}(\mathcal{D}) M^{2}
$$

Corollary 1. Let $\mathcal{D}$ be a planar domain whose boundary $C=\partial \mathcal{D}$ satisfies the conditions stated above. Then it follows that, for $X$ large,

$$
\int_{0}^{X}\left(\mathcal{R}_{D}(M)\right)^{2} \mathrm{~d} M \ll X^{2} .
$$

If, moreover, the pieces of the boundary $\mathcal{C}_{j}$ are either all convex or all concave with respect to the domain $\mathcal{D}$, then also

$$
\lim _{M \rightarrow \infty} \sup \left(\frac{ \pm \mathcal{R}_{\mathcal{D}}(M)}{M^{1 / 2}(\log M)^{1 / 4}}\right)>0
$$

where \pm depends on whether the $\mathcal{C}_{j}$ are concave $(+)$ or convex $(-)$.

Remarks. 1. The sharpest known upper bound for the discrepancy of a domain $\mathcal{D}$ satisfying the conditions of Corollary 1 was established in the deep paper of Huxley [11] and reads

$$
\mathcal{R}_{\mathcal{D}}(M) \ll M^{46 / 73}(\log M)^{315 / 146}
$$

2. In the classic literature, lower bounds and mean-square results for the lattice rest of circles and ellipsoids were usually derived by means of the functional equation of the Epstein zeta-function of a positive definite quadratic form. These results in turn can be seen as special cases of much more general theorems of this kind: see, e.g., Chandrasekharan-Narasimhan [3], Redmond [23], respectively Hafner [8] and the references cited there.

Not too much different is the case of a domain $\mathcal{D}$ whose boundary $\mathcal{C}$ is of class $C^{\infty}$ throughout: Here $\mathcal{D}$ possesses a so-called Hlawka zeta-function (see Hlawka [9]) which shares some characteristic properties with an Epstein zeta-function. By methods essentially based on this fact, the author $[21,22]$ had established earlier the results of Corollary 1 for such convex sets $\mathcal{D}$ with $C^{\infty}$-boundary.

The novelty of the present paper is thus (apart from replacing $C^{\infty}$ by $C^{4}$ ) that the curve $C$ now may have corners or may even possess an asteroid-like shape. E.g., for the domain

$$
\mathcal{D}_{0}: \quad \sqrt{|\xi|}+\sqrt{|\eta|} \leq 1
$$


our results imply that

$$
\mathcal{R}_{\mathcal{D}_{0}}(M)=\Omega_{+}\left(M^{1 / 2}(\log M)^{1 / 4}\right), \quad \int_{0}^{X}\left(\mathcal{R}_{\mathcal{D}_{0}}(M)\right)^{2} \mathrm{~d} M \ll X^{2} .
$$

For this very special case, it is known [20] that

$$
\mathcal{R}_{D_{0}}(M) \ll M^{1 / 2} \exp \left(\frac{c \log M}{\log \log M}\right) \quad(c>0) .
$$

3. For $\mathcal{D}$ with smooth $C^{4}$-boundary throughout, Huxley [12] has obtained recently a mean-square estimate for a much shorter interval, i.e.,

$$
\int_{X}^{X+1}\left(\mathcal{R}_{\mathcal{D}}(M)\right)^{2} \mathrm{~d} M \ll X \log X
$$

It is likely that further work could yield this bound under the general conditions of Theorem 2.

4. As a consequence of the freedom that (1.1) provides concerning the values of $\psi$ at the integers, we may count lattice points on the boundary of $M D$ with any weight $\omega \in[0,1]$, without affecting the validity of our results. This is obvious for the meansquare estimate (since the $M$-values for which $M \mathcal{D}$ contains any lattice points form a set of measure zero) but perhaps remarkable for the $\Omega$-bound: It should be observed that all we know in general about the total number of lattice points on $M D$ is that it is $\ll M^{3+\varepsilon}$, for any $\varepsilon>0$, according to a deep work of Swinnerton-Dyer [24].

Proof of Corollary 1. We shall show that $\mathcal{R}_{D}(M)$ can be represented in the form $-\mathcal{S}(M)+O(1)$, with $\mathcal{S}(M)$ as in Section 1 . We start with one of the curves $\mathcal{C}_{j}$ with endpoints $\left(\alpha_{1}, \beta_{1}\right)$ and $\left(\alpha_{2}, \beta_{2}\right)$ (with $\frac{\beta_{1}}{\alpha_{1}}<\frac{\beta_{2}}{\alpha_{2}}$ ), both contained in the interior of the first quadrant. We may assume that $\mathcal{C}_{j}$ is in Cartesian coordinates $(\xi, \eta)$ the graph of a $C^{4}$-function $\eta=f_{j}(\xi)$ (or vice-versa); if necessary, we might refine the subdivision of $[0,2 \pi]$. Now let $\mathcal{D}_{j}$ denote the sector which is bounded by the curve $\mathcal{C}_{j}$ and the two straight line segments joining the origin with $\left(\alpha_{1}, \beta_{1}\right),\left(\alpha_{2}, \beta_{2}\right)$, respectively. Let further $N_{\mathcal{D}_{j}}(M)$ denote the number of lattice points in $M \mathcal{D}_{j}$ where points on the straight line segments are weighted with the factor $\frac{1}{2}$ while points on $M C_{j}$ get any weight $\omega \in[0,1]$. Then an elementary lattice point counting argument shows that

$$
N_{\mathcal{D}_{j}}(M)=\operatorname{area}\left(\mathcal{D}_{j}\right) M^{2}-\Psi_{\left(\alpha_{2}, \beta_{2}\right)}(M)+\Psi_{\left(\alpha_{1}, \beta_{1}\right)}(M)-e_{j} S_{j}(M)+O(1)
$$

where

$$
\Psi_{(\alpha, \beta)}(M)=\sum_{1 \leq n \leq \alpha M} \psi_{0}\left(\frac{\beta}{\alpha} n\right), \quad \psi_{0}(t)= \begin{cases}t-[t]-\frac{1}{2} & \text { for } t \notin \mathbb{Z} \\ 0 & \text { for } t \in \mathbb{Z}\end{cases}
$$


$S_{j}(M)$ is a $\psi$-sum as defined in section 1 , and $e_{j}=+1$ if $\mathcal{D}$ is below the curve $\mathcal{C}_{j}$ and $e_{j}=-1$ if $\mathcal{D}$ is above $\mathcal{C}_{j}$.

If $\mathcal{C}_{j}$ happens to have a vertical tangent and thus can be represented only in the form $\xi=f_{j}^{*}(\eta)$, we may interchange the rôles of the variables and then apply the elementary identity

$$
\Psi_{(\alpha, \beta)}(M)=\Psi_{(\beta, \alpha)}(M)+O(1)
$$

to obtain (2.1) with the same $\Psi$ 's. Further, if $\left(\alpha_{1}, \beta_{1}\right)$ lies on the horizontal or $\left(\alpha_{2}, \beta_{2}\right)$ on the vertical axis, the corresponding $\Psi$-term vanishes. Putting together adjacent sectors $\mathcal{D}_{j}$ we see that the $\Psi$-expressions cancel and obtain

$$
N_{D}^{(1)}(M)=\sum_{j} e_{j} S_{j}(M)+O(1)
$$

where $N_{\mathcal{D}}^{(1)}(M)$ denotes the number of lattice points of $M \mathcal{D}$ which lie in the first quadrant, points on the axes being counted with weight $\frac{1}{2}$. By suitable transformations, the same argument applies to the other quadrants. (Note that the substitution $\xi \rightarrow-\xi$ leaves invariant the sign of $f_{j}^{\prime \prime}$, and that $-\psi(-t)$ again meets the conditions (1.1) if $\psi(t)$ does.) Finally, a little reflection shows that the convexity conditions together with the factors $e_{j}$ just ensure that the requirements concerning the second derivatives of Theorem 1 are satisfied. This completes the proof of Corollary 1.

The analysis just carried out enables us to apply our estimates to the case that $\mathcal{D}$ is a rather general sort of sector. Let

$$
\mathcal{C}: \quad r=\rho\left(\lambda_{)}, \quad \lambda_{0} \leq \lambda \leq \lambda_{J},\right.
$$

$\rho$ continuous on $\left[\lambda_{0}, \lambda_{J}\right]$, with a subdivision

$$
0 \leq \lambda_{0}<\lambda_{1}<\ldots<\lambda_{s} \leq 2 \pi
$$

such that on each subinterval $\left[\lambda_{j-1}, \lambda_{j}\right]$ all the conditions stated earlier are satisfied.

Let

$$
P_{1}=\left(\rho\left(\lambda_{0}\right) \cos \lambda_{0}, \rho\left(\lambda_{0}\right) \sin \lambda_{0}\right), \quad P_{2}=\left(\rho\left(\lambda_{J}\right) \cos \lambda_{J}, \rho\left(\lambda_{J}\right) \sin \lambda_{J}\right)
$$

be the endpoints of $\mathcal{C}$, and call $\mathcal{D}$ the planar domain bounded by $\mathcal{C}$ and the two straight line segments from the origin to $P_{1}, P_{2}$, respectively. Then the above argument gives for $N_{\mathcal{D}}(M)$ immediately a representation $(2.1)$ involving $\Psi_{P_{1}}(M), \Psi_{P_{2}}(M)$. In order that these do not affect our estimates, it is desirable that they should be $\ll M^{1 / 2}$. For $P=(\alpha, \beta)$ with $\beta / \alpha$ rational this is no difficulty: A short elementary calculation shows that

$$
\Psi_{(x, \beta)}(M) \ll 1 \quad \text { for fixed rational } \frac{\beta}{\alpha}
$$


For irrational $\beta / \alpha$ we have to recall a few facts from the theory of Diophantine approximation: By the (approximation) type $t(\gamma)$ of an irrational real number $\gamma$ we understand the infimum of all reals $r$ for which there exists a constant $c(r, \gamma)>0$ such that

$$
\left|\gamma-\frac{p}{q}\right| \geq \frac{c(r, \gamma)}{q^{r+1}}
$$

for all integers $p$ and all positive integers $q$. We note that, according to a celebrated result of Thue, Siegel, and Roth, all algebraic irrationals $\gamma$ are of type $t(\gamma)=1$. By another well-known (though less deep) theorem due to Khintchin, the same is true of almost all reals $\gamma$ (in the sense of Lebesgue).

We use the following bound:

$$
\sum_{1 \leq n \leq x M} \psi_{0}(\gamma n) \ll M^{1-1 /(\gamma)+\varepsilon} \quad(\varepsilon>0)
$$

for fixed $\alpha>0$ and fixed algebraic $\gamma$. This is apparently essentially due to Hardy and can be found in convenient form in the book of Kuipers-Niederreiter [18, p. 123 and 143]. For our purpose, this implies that

$$
\Psi_{(\alpha, \beta)}(M) \ll M^{1 / 2} \quad \text { if } t\left(\frac{\beta}{\alpha}\right)<2 .
$$

We may thus summarize our conclusions as follows.

Corollary 2. Let $\mathcal{D}$ be a general sector, bounded by a piecewise smooth curve $\mathcal{C}$ with endpoints $P_{1}=\left(\alpha_{1}, \beta_{1}\right), P_{2}=\left(\alpha_{2}, \beta_{2}\right)$, and the straight line segments $O P_{1}, O P_{2}$. Suppose that $\mathcal{C}$ is composed of finitely many pieces $\mathcal{C}_{j}$ (as described above) each of which is of class $C^{4}$, has finite nonzero curvature throughout, and satisfies the tangent condition. Assume further that $\frac{\beta_{1}}{\alpha_{1}}$ and $\frac{\beta_{2}}{\alpha_{2}}$ are either rational or irrational of an approximation type less than 2 . Then the lattice rest $\mathcal{R}_{\mathcal{D}}(M)$ satisfies

$$
\int_{0}^{X}\left(\mathcal{R}_{\mathcal{D}}(M)\right)^{2} \mathrm{~d} M \ll X^{2}
$$

If, in addition, the pieces $\mathcal{C}_{j}$ are either all convex or all concave with respect to the domain $\mathcal{D}$, then also

$$
\lim _{M \rightarrow \infty} \sup \left(\frac{ \pm \mathcal{R}_{\mathcal{D}}(M)}{M^{1 / 2}(\log M)^{1 / 4}}\right)>0
$$

where \pm depends on whether the $\mathcal{C}_{j}$ are concave $(+)$ or convex $(-)$. 


\section{Some lemmas}

Lemma 1. (Transition from fractional parts to trigonometric polynomials according to Vaaler [25].) For arbitrary $w \in \mathbb{R}$ and $H \in \mathbb{N}$, suppose that $\psi$ satisfies (1.1), and put

$$
\psi_{H}^{*}(w)=-\frac{1}{\pi} \sum_{h=1}^{H} \frac{\sin (2 \pi h w)}{h} \tau\left(\frac{h}{H+1}\right)
$$

where

$$
\tau(\xi)=\pi \xi(1-\xi) \cot (\pi \xi)+\xi \quad \text { for } 0<\xi<1 .
$$

Then there holds the inequality

$$
\left|\psi(w)-\psi_{H}^{*}(w)\right| \leq \frac{1}{H+1} \sum_{h=1}^{H}\left(1-\frac{h}{H+1}\right) \cos (2 \pi h w)+\frac{1}{2 H+2} .
$$

Proof. For $w \notin \mathbb{Z}$, this is one of the main results in Vaaler [25]. For a very well readable exposition, see also the book of Graham and Kolesnik [6, p. 116]. The case $w \in \mathbb{Z}$ is an obvious consequence by a limit argument or by direct evaluation.

Lemma 2. For a real parameter $P \geq 1$, let $F_{\mathrm{P}}$ denote the Fejér kernel

$$
F_{P}(w)=P\left(\frac{\sin (\pi P w)}{\pi P w}\right)^{2}
$$

Then for arbitrary real $Q \neq 0, \gamma \in \mathbb{R}$, it follows that

$$
\int_{-1}^{1} F_{P}(w) \cos (2 \pi Q w+\gamma) \mathrm{d} w=\max \left(1-\frac{|Q|}{P}, 0\right) \cos \gamma+O\left(\frac{1}{|Q|}\right)
$$

where the $O$-constant is independent of $P$ and $\gamma$.

Proof. This is adopted from Hafner [7]. The reader may recall the classic Fourier transform formula

$$
\int_{\mathbf{R}} F_{P}(w) \exp (2 \pi i Q w) \mathrm{d} w=\int_{\mathbf{R}}\left(\frac{\sin (\pi w)}{\pi w}\right)^{2} \exp \left(2 \pi i \frac{|Q|}{P} w\right) \mathrm{d} w=\max \left(1-\frac{|Q|}{P}, 0\right)
$$

Since $F_{P}( \pm 1)=O\left(P^{-1}\right)$ and $F_{P}^{\prime}(w)=O\left(w^{-2}\right)$ for $|w| \geq 1$ (uniformly in $P \geq 1$ ), integration by parts readily shows that the intervals $]-\infty,-1]$ and $[1, \infty[$ contribute only $O\left(|Q|^{-1}\right)$. Multiplying by $\exp (i \gamma)$ and taking the real part on both sides gives the assertion. 
Lemma 3. (A strong form of the van der Corput transform.) Let $f$ be a real function with continuous derivatives up to the fourth order on an interval $[a, b]$. Suppose that $f^{\prime \prime}(w)<0$ on $[a, b]$ throughout. Let $h \geq 1, U \geq 1$, be real parameters. Let finally $\varphi$ denote the inverse function of $f^{\prime}$, and write $e(\xi)$ for $e^{2 \pi i \xi}$ as usual. Then it follows that

$$
\begin{aligned}
\sum_{a U<n \leq b U} e\left(-h U f\left(\frac{n}{U}\right)\right)= & e\left(\frac{1}{8}\right) \sqrt{\frac{U}{h}} \sum_{-h f^{\prime}(a) \leq m \leq-h f^{\prime}(b)} \frac{e(-U G(m, h))}{\sqrt{\left|f^{\prime \prime}\left(\varphi\left(-\frac{m}{h}\right)\right)\right|}} \\
& +o\left(\sqrt{\frac{U}{h}}\right)+O(\log (1+h))
\end{aligned}
$$

where

$$
G(u, v)=v f\left(\varphi\left(-\frac{u}{v}\right)\right)+u \varphi\left(-\frac{u}{v}\right)
$$

Proof. This is an immediate consequence of Lemma 3.6 in Graham and Kolesnik [6]. We are going to have a closer look at the function $G(u, v)$ : Its natural domain of definition is

$$
\mathcal{D}^{*}=\left\{(u, v) \in \mathbb{R}^{2}: v>0,-v f^{\prime}(a) \leq u \leq-v f^{\prime}(b)\right\}
$$

We shall construct a map $\mathcal{M}: \mathcal{D}^{*} \rightarrow \mathcal{C}$ where $\mathcal{C}$ is the curve

$$
\mathcal{C}: \quad \eta=f(\xi), \quad a \leq \xi \leq b .
$$

For given $(u, v) \in \mathcal{D}^{*},(\xi, \eta)=\mathcal{M}(u, v)$ is defined as that point of the curve $\mathcal{C}$ where the vector $(u, v)$ is orthogonal to $\mathcal{C}$. Then the distance of the origin from the tangent of $\mathcal{C}$ in $(\xi, \eta)=\mathcal{M}(u, v)$ is equal to

$$
\frac{|G(u, v)|}{\sqrt{u^{2}+v^{2}}} .
$$

For what follows, we shall assume that the curve $\mathcal{C}$ satisfies the tangent condition. As a first consequence, it is clear that $|G(u, v)|$ attains a positive minimum and maximum on

$$
\left\{(u, v) \in \mathcal{D}^{*}: \quad u^{2}+v^{2}=1\right\}
$$

Since $G(u, v)$ is homogeneous of order 1 , it follows that there exist constants $c_{2}>c_{1}>0$, such that, for all $(u, v) \in \mathcal{D}^{*}$,

$$
c_{1} \sqrt{u^{2}+v^{2}} \leq|G(u, v)| \leq c_{2} \sqrt{u^{2}+v^{2}}
$$


It follows further from the tangent condition that $G(u, v)$ is of the same sign throughout $\mathcal{D}^{*}$ : let $e^{*} \in\{-1,1\}$ denote this sign. We consider the curve

$$
\mathcal{C}^{*}=\left\{(u, v) \in \mathcal{D}^{*}: \quad G(u, v)=e^{*}\right\}
$$

Its endpoints are

$$
\left(\frac{-f^{\prime}(a) e^{*}}{f(a)-a f^{\prime}(a)}, \frac{e^{*}}{f(a)-a f^{\prime}(a)}\right) \text { and }\left(\frac{-f^{\prime}(b) e^{*}}{f(b)-b f^{\prime}(b)}, \frac{e^{*}}{f(b)-b f^{\prime}(b)}\right)
$$

Denote by $\mathcal{M}_{0}$ the restriction of $\mathcal{M}$ to $\mathcal{C}^{*}$, then it is easy to see that $\mathcal{M}_{0}: \mathcal{C}^{*} \rightarrow \mathcal{C}$ is one-one. Further, if $\rho^{*}(u, v)$ is the radius of curvature of $\mathcal{C}^{*}$ at $(u, v)$, and $\rho(\xi, \eta)$ that of $\mathcal{C}$ at the corresponding point $(\xi, \eta)=\mathcal{M}_{0}(u, v)$, a recent and elaborate result of Huxley $[12$, Lemma 4], tells us that

$$
\rho(\xi, \eta) \rho^{*}(u, v) \sin ^{3} \lambda(u, v)=1
$$

where $\lambda(u, v)$ is the angle between the radius vector $\left(\begin{array}{l}\xi \\ \eta\end{array}\right)$ and the tangent of $\mathcal{C}$ in the point $(\xi, \eta)$. (A multi-dimensional analogue of this formula has been established quite recently by Müller [19].) Because of the tangent condition, $\lambda(u, v)$ is bounded away from 0 and $\pi$. Therefore, since $\mathcal{C}$ has finite nonzero curvature throughout, the same is true for the curve $C^{*}$. This enables us to estimate the number of lattice points in the strip between the curves $Y C^{*}$ and $(Y-\Delta) \mathcal{C}^{*}$ where $Y$ is large and $0<\Delta \leq \frac{1}{2} Y$ :

Lemma 4. Suppose that the function $f$ satisfies the conditions of our theorems, and let $N(Y, \Delta)$ denote the number of lattice points in

$$
\left\{(u, v) \in \mathbb{R}^{2}: \quad Y-\Delta \leq e^{*} G(u, v) \leq Y, v>0,-v f^{\prime}(a) \leq u \leq v f^{\prime}(b)\right\} .
$$

Then it follows that

$$
N(Y, \Delta) \ll Y^{2 / 3}+\Delta Y
$$

Proof. For $\Delta \geq \frac{1}{4}$ (say) this is trivial, since

$$
N(Y, \Delta) \ll \text { area }+ \text { length of the boundary }
$$

gives what we need. For $\Delta<\frac{1}{4}$, we may appeal to a result of Branton and Sargos [2] and Huxley and Sargos [14].

\section{Proof of Theorem 1}

We may suppose that $f_{j}^{\prime \prime}(w)<0$ throughout. Otherwise we might replace all $f_{j}$ by $-f_{j}$, since $-\psi_{j}(-t)$ again satisfies the requirements (1.1). For what follows, let $M$ be a 
large real parameter, and let $U \in[M-1, M+1]$. Our first important step is to apply Lemma 1 in order to compare the fractional part sum with an appropriate trigonometric polynomial. We choose $H=[M]$ and infer from Lemma 1 that, for any $w \in \mathbb{R}$,

$$
\psi(w) \geq-\sum_{1 \leq h \leq M}\left(\frac{1}{\pi h} \tau\left(\frac{h}{[M]+1}\right) \sin (2 \pi h w)+\frac{1-\frac{h}{[M]+1}}{[M]+1} \cos (2 \pi h w)\right)-\frac{1}{2[M]+2} .
$$

Consequently,

$$
\begin{aligned}
\mathcal{S}(U) \geq & \sum_{j=1}^{J} \sum_{1 \leq h \leq M}\left(\frac{1}{\pi h} \tau\left(\frac{h}{[M]+1}\right) \sum_{a_{j} U<n \leq b_{j} U} \sin \left(-2 \pi h U f_{j}\left(\frac{n}{U}\right)\right)\right. \\
& \left.-\frac{1-\frac{h}{[M]+1}}{[M]+1} \sum_{a_{j} U<n \leq b_{j} U} \cos \left(-2 \pi h U f_{j}\left(\frac{n}{U}\right)\right)\right)-C_{0} .
\end{aligned}
$$

Our next step is to transform each of the inner trigonometric sums over $n$ by Lemma 3. We start with estimating the contribution of the $O$-terms from the Lemma: This is

$$
\ll \sum_{1 \leq h \leq M} \frac{1}{h}\left(\frac{M^{1 / 2}}{\sqrt{h}}+\log M\right) \ll M^{1 / 2}
$$

since $\tau$ is bounded. Using the real and imaginary part of Lemma 3 separately, we thus conclude that

$$
\mathcal{S}(U) \geq U^{1 / 2} \sum_{j=1}^{J} S_{j}^{*}(U)-C_{1} U^{1 / 2}
$$

where

$$
S_{j}^{*}(U)=\sum_{(m, h) \in \mathcal{D}_{j}(M)} \frac{\beta_{1}(h, M) \sin \left(-2 \pi U G_{j}(m, h)+\frac{\pi}{4}\right)-\beta_{2}(h, M) \cos \left(-2 \pi U G_{j}(m, h)+\frac{\pi}{4}\right)}{\sqrt{\left|f_{j}^{\prime \prime}\left(\varphi_{j}\left(\frac{-m}{h}\right)\right)\right|}}
$$

with

$$
\begin{gathered}
\beta_{1}(h, M)=\frac{1}{\pi h^{3 / 2}} \tau\left(\frac{h}{[M]+1}\right), \quad \beta_{2}(h, M)=\frac{1-\frac{h}{[M]+1}}{h^{1 / 2}([M]+1)} \\
G_{j}(m, h)=h f_{j}\left(\varphi_{j}\left(-\frac{m}{h}\right)\right)+m \varphi_{j}\left(-\frac{m}{h}\right)
\end{gathered}
$$


for short ( $\varphi_{j}$ the inverse function of $f_{j}^{\prime}$ ), and the domain of summation $\mathcal{D}_{j}(M)$ is

$$
\mathcal{D}_{j}(M)=\left\{(m, h) \in \mathbb{Z}^{2}: \quad 1 \leq h \leq M,-h f_{j}^{\prime}(a) \leq m \leq-h f_{j}^{\prime}(b)\right\} .
$$

We would like to estimate $S_{j}^{*}(U)$ from below. However, the trigonometric sum involved still contains too many terms. To get rid of the greater part of them, we multiply $S_{j}^{*}(U)$ by a Fejér kernel $F_{P}(U-M)$ (where $P$ is a new large real parameter) and integrate over $U$ from $M-1$ to $M+1$. In view of Lemma 2, we thus obtain

$$
\begin{aligned}
I_{j}(M):= & \int_{M-1}^{M+1} S_{j}^{*}(U) F_{P}(U-M) \mathrm{d} U=\int_{-1}^{1} S_{j}^{*}(M+w) F_{P}(w) \mathrm{d} w \\
= & \sum_{\substack{(m, h) \in \mathcal{D},(M): \\
\mid G_{j}(m, h) \leq P}}\left(1-\frac{\left|G_{j}(m, h)\right|}{P}\right)\left(\frac{\beta_{1}(h, M)}{\kappa_{j}(m, h)} \sin \left(-2 \pi M G_{j}(m, h)+\frac{\pi}{4}\right)\right. \\
& \left.-\frac{\beta_{2}(h, M)}{\kappa_{j}(m, h)} \cos \left(-2 \pi M G_{j}(m, h)+\frac{\pi}{4}\right)\right)+\sum_{(m, h) \in \mathcal{D}_{j}(M)} O\left(h^{-3 / 2}\left|G_{j}(m, h)\right|^{-1}\right),
\end{aligned}
$$

with $\kappa_{j}(m, h)=\sqrt{\left|f_{j}^{\prime \prime}\left(\varphi_{j}\left(\frac{-m}{h}\right)\right)\right|} \approx 1$. (For the $O$-term, (4.3) has been used.) Let us estimate first the remainder term sum: By (3.1),

$$
\begin{aligned}
& \sum_{(m, h) \in \mathcal{D}_{j}(M)} O\left(h^{-3 / 2}\left|G_{j}(m, h)\right|^{-1}\right) \ll \sum_{(m, h) \in \mathcal{D}_{j}(M)} h^{-5 / 2} \\
= & \sum_{1 \leq h \leq M} h^{-5 / 2} \sum_{-h f_{j}^{\prime}(a) \leq m \leq-h f_{j}^{\prime}(b)} 1 \ll \sum_{1 \leq h \leq M} h^{-3 / 2} \ll 1 .
\end{aligned}
$$

The next important step is to make sure that in the sum which represents the main portion of $I_{j}(M)$ all terms are positive. For that purpose we specify the relation between the two parameters $P$ and $M$ involved: For given $P$ sufficiently large, we pick $M$ according to Dirichlet's approximation theorem such that

$$
\left\|M G_{j}(m, h)\right\| \leq \frac{1}{16} \quad \text { for all }(m, h) \in \mathbb{Z}^{2} \text { with }\left|G_{j}(m, h)\right| \leq P
$$

and for all $j=1, \ldots, J$ (where $\|$.$\| denotes the distance from the nearest integer), and$

$$
P^{2} \leq M \leq P^{2} 16^{c_{3} P^{2}}
$$

(By (3.1), for each $j$ the number of integer pairs $(m, h)$ with $\left|G_{j}(m, h)\right| \leq P$ is $\ll P^{2}$.) As an immediate consequence of (i),

$$
\sin \left(-2 \pi M G_{j}(m, h)+\frac{\pi}{4}\right) \geq \sin \frac{\pi}{8} \quad \text { and } \quad-\cos \left(-2 \pi M G_{j}(m, h)+\frac{\pi}{4}\right) \geq-\cos \frac{\pi}{8}
$$


for all $(m, h)$ involved in the sum. Furthermore, again by (3.1),

$$
h \leq \sqrt{m^{2}+h^{2}} \ll\left|G_{j}(m, h)\right| \leq P \leq \sqrt{M},
$$
thus it is clear that, for $P$ large, $h^{3 / 2} \beta_{1}(h, M)$ is close to $\frac{1}{\pi}$ throughout, (observing that
$\tau(\xi) \rightarrow 1$ as $\xi \rightarrow 0)$, and $h^{3 / 2} \beta_{2}(h, U)$ is small, hence

$$
h^{3 / 2}\left(\frac{\beta_{1}(h, M)}{\kappa_{j}(m, h)} \sin \left(-2 \pi M G_{j}(m, h)+\frac{\pi}{4}\right)-\frac{\beta_{2}(h, M)}{\kappa_{j}(m, h)} \cos \left(-2 \pi M G_{j}(m, h)+\frac{\pi}{4}\right)\right) \geq C_{2}>0
$$

for all $(m, h) \in \mathcal{D}_{j}(M)$ with $\left|G_{j}(m, h)\right| \leq P$, and for all $j=1, \ldots, J$. We now choose $c_{4}>0$ sufficiently small and put

$$
\mathcal{D}_{j}^{* *}(P)=\left\{(m, h) \in \mathbb{Z}^{2}: \quad 1 \leq h \leq c_{4} P,-h f_{j}^{\prime}(a) \leq m \leq-h f_{j}^{\prime}(b)\right\},
$$

then it follows that

$$
I_{j}(M) \geq C_{3} \sum_{(m, h) \in D_{j}^{-*}(P)} h^{-3 / 2}-C_{4}
$$

Now

$$
\begin{aligned}
& \sum_{(m, h) \in \mathcal{D}_{j}^{+*}(P)} h^{-3 / 2}=\sum_{1 \leq h \leq c_{4} P} h^{-3 / 2} \sum_{-h f_{j}^{\prime}(a) \leq m \leq-h j_{j}^{\prime}(b)} 1 \\
& \geq C_{5} \sum_{1 \leq h \leq c_{4} P} h^{-1 / 2} \geq C_{6} P^{1 / 2} \geq C_{7}(\log M)^{1 / 4},
\end{aligned}
$$

where the very last inequality is a consequence of (ii). Thus, for $j=1, \ldots, J$,

$$
I_{j}(M) \geq C_{8}(\log M)^{1 / 4} .
$$

On the other hand, by the definition of $I_{j}(M)$,

$$
\sum_{j=1}^{J} I_{j}(M) \leq \sup _{M-1 \leq U \leq M+1}\left(\sum_{j=1}^{J} S_{j}^{*}(U)\right) \int_{-1}^{1} F_{P}(w) \mathrm{d} w .
$$

Since

$$
\int_{-1}^{1} F_{P}(w) \mathrm{d} w=\int_{-P}^{p}\left(\frac{\sin (\pi w)}{\pi w}\right)^{2} \mathrm{~d} w \leq 1
$$

there exists some $U^{*} \in[M-1, M+1]$ such that

$$
\sum_{j=1}^{J} S_{j}^{*}(U) \geq C_{9}\left(\log U^{*}\right)^{1 / 4}
$$


It remains to recall that if $P$ runs through an unbounded sequence of values, so do $M$ and $U^{*}$ by construction, in particular in view of (ii). Therefore, (4.5) together with (4.2) completes the proof of Theorem 1.

\section{Proof of Theorem 2}

We may assume that $J=1$ and thus have to estimate

$$
\mathcal{I}(X)=\int_{X}^{2 X}(S(M))^{2} \mathrm{~d} M
$$

It is easy to see from Lemma 1 that there exists a sequence of complex numbers $(\gamma(h, X))_{1 \leq h \leq X}$ with

$$
\gamma(h, X) \ll \frac{1}{h}
$$

such that

$$
\mathcal{I}(X) \ll \int_{X}^{2 X}\left|\sum_{1 \leq h \leq X} \gamma(h, X) \sum_{a M<n \leq b M, n \in \mathbf{Z}} e\left(-h M f\left(\frac{n}{M}\right)\right)\right|^{2} \mathrm{~d} M+X
$$

Assuming again that $f^{\prime \prime}<0$, we infer from Lemma 3 that

$$
\sum_{a M<n \leq b M} e\left(-h M f\left(\frac{n}{M}\right)\right)=e\left(\frac{1}{8}\right) \sqrt{\frac{M}{h}} \sum_{-h f^{\prime}(a) \leq m \leq h f^{\prime}(b)} \frac{e(-M G(m, h))}{\sqrt{\left|f^{\prime \prime}\left(\varphi\left(-\frac{m}{h}\right)\right)\right|}}+O\left(\sqrt{\frac{M}{h}}\right)+O(\log X) .
$$

We first estimate the contribution of the $O$-terms: It is clear that

$$
\int_{X}^{2 X}\left(\sum_{h \leq X} \frac{1}{h}\left(\frac{\sqrt{M}}{\sqrt{h}}+\log X\right)\right)^{2} \mathrm{~d} M \ll X^{2}
$$

Therefore, by Cauchy's inequality,

$$
\mathcal{I}(X) \ll X \mathcal{I}^{*}(X)+X^{3 / 2} \mathcal{I}^{*}(X)^{1 / 2}+X^{2}
$$

where

$$
\mathcal{I}^{*}(X)=\int_{X}^{2 X}\left|\sum_{h \leq X} \Sigma(h, M)\right|^{2} \mathrm{~d} M
$$




$$
\begin{gathered}
\Sigma(h, M)=\frac{\gamma(h, X)}{\sqrt{h}} \sum_{-h f^{\prime}(a) \leq m \leq-h^{\prime}(b)} \frac{e(-M G(m, h))}{\kappa(m, h)}, \\
\kappa(m, h)=\sqrt{\left|f^{\prime \prime}\left(\varphi\left(-\frac{m}{h}\right)\right)\right|} \asymp 1 .
\end{gathered}
$$

We split up the domain of summation over $h$ into dyadic subintervals: Let

$$
\left.\left.\mathbb{H}_{i}=\right] H_{i+1}, H_{i}\right], \quad H_{i}=\frac{X}{2^{i}}, \quad i=0,1, \ldots, I,
$$

where $I$ is the largest integer for which $2^{I}<X$. By Cauchy's inequality,

$$
\left|\sum_{h \leq X} \Sigma(h, M)\right|^{2}=\left|\sum_{i=0}^{I} \sum_{h \in \mathbf{H}_{i}} \Sigma(h, M)\right|^{2} \leq \sum_{i=0}^{I} H_{i}^{-\varepsilon} \sum_{i=0}^{I} H_{i}^{e}\left|\sum_{h \in \mathbf{H}_{i}} \Sigma(h, M)\right|^{2} \ll \sum_{i=0}^{I} H_{i}^{e}\left|\sum_{h \in \mathbf{H}_{i}} \Sigma(h, M)\right|^{2},
$$

with some fixed $\varepsilon>0$ sufficiently small. Therefore,

$$
\mathcal{I}^{*}(X) \ll \sum_{i=0}^{1} H_{i}^{e} \mathcal{I}_{i}^{*}(X)
$$

with

$$
\mathcal{I}_{i}^{*}(X)=\int_{X}^{2 X}\left|\sum_{h \in \mathbf{H}_{i}} \Sigma(h, M)\right|^{2} \mathrm{~d} M
$$

In what follows, we write $\mathbf{u}=\left(u_{1}, u_{2}\right), \mathbf{v}=\left(v_{1}, v_{2}\right)$ for elements of $\mathbb{Z}^{2}$, and put

$$
\mathcal{A}_{i}=\left\{(m, h) \in \mathbb{Z}^{2}: \quad h \in \mathbb{H}_{i},-h f^{\prime}(a) \leq m \leq-h f^{\prime}(b)\right\} .
$$

A straightforward calculation yields

$$
\begin{aligned}
\mathcal{I}_{i}^{*}(X) & =\sum_{\mathbf{a}, v \in \mathcal{A}_{i}} \frac{\gamma\left(u_{2}, X\right) \gamma\left(v_{2}, X\right)}{\sqrt{u_{2} v_{2}} \kappa(\mathbf{u}) \kappa(\mathbf{v})} \int_{X}^{2 X} e(-M(G(\mathbf{u})-G(\mathbf{v}))) \mathrm{d} M \\
& \ll \sum_{\mathbf{u}, v \in \mathcal{A}_{i}}\left(u_{2} v_{2}\right)^{-3 / 2} \min \left(X, \frac{1}{|G(\mathbf{u})-G(\mathbf{v})|}\right) \\
& \ll H_{i}^{-3} \sum_{\mathbf{u} \in \mathcal{A}_{i}}\left(\sum_{\substack{v \in \mathcal{A}_{i} \\
: G(v) \leq e^{\circ}(\mathbf{v})}} \min \left(X, \frac{1}{e^{*}(G(\mathbf{u})-G(\mathbf{u}))}\right)\right)
\end{aligned}
$$

with an appeal to the bound (5.1) for the coefficients $\gamma(\cdot)$. 
We now keep $u \in \mathcal{A}_{i}$ fixed for the moment and split up the inner sum over v. First of all,

$$
X<\frac{1}{e^{*} G(\mathbf{u})-e^{*} G(\mathbf{v})} \Leftrightarrow e^{*} G(\mathbf{u})-\frac{1}{X}<e^{*} G(\mathbf{v}) \leq e^{*} G(\mathbf{u})
$$

In view of Lemma 4 , the total contribution of these $v$ to the inner sum in (5.5) is thus

$$
\ll X N\left(e^{*} G(\mathbf{u}), \frac{1}{X}\right) \ll X\left(e^{*} G(\mathbf{u})\right)^{2 / 3}+e^{*} G(\mathbf{u})
$$

To deal with the other v's, we define a sequence $\left(\delta_{r}\right)_{r=0}^{R}$, by $\delta_{r}=2^{r} / X$ for $r=0,1, \ldots, R-1$, and $\delta_{R}=e^{*} G(\mathrm{u})$, where $R$ is the largest integer such that $2^{R} / X<e^{*} G(\mathbf{u})$. Then

$$
e^{*} G(\mathbf{u})-e^{*} G(\mathbf{v}) \in\left[\delta_{r}, \delta_{r+1}\left[\Leftrightarrow e^{*} G(\mathbf{u})-\delta_{r+1}<e^{*} G(\mathbf{v}) \leq e^{*} G(\mathbf{u})-\delta_{r},\right.\right.
$$

thus, again by Lemma 4, the corresponding portion of the inner sum in (5.5) is

$$
\ll \frac{1}{\delta_{r}} N\left(e^{*} G(\mathbf{u})-\delta_{r}, \delta_{r}\right) \ll \frac{1}{\delta_{r}}\left(e^{*} G(\mathbf{u})\right)^{2 / 3}+e^{*} G(\mathbf{u})
$$

Summing this over $r=0,1, \ldots, R-1$ and adding (5.6), we obtain altogether

$$
\sum_{\mathbf{v} \in \mathcal{A}_{i}: e^{*} G(\mathbf{v}) \leq e^{*} G(\mathbf{u})} \min \left(X, \frac{1}{e^{*} G(\mathbf{u})-e^{*} G(\mathbf{v})}\right) \ll X\left(e^{*} G(\mathbf{u})\right)^{2 / 3}+e^{*} G(\mathbf{u}) \log X .
$$

By (3.1) and the definition of $\mathcal{A}_{i}$, it follows that $e^{*} G(\mathbf{u}) \ll H_{i}$ for $\mathbf{u} \in \mathcal{A}_{i}$. Therefore, inserting (5.7) into (5.5), we arrive at

$$
\mathcal{I}_{i}^{*}(X) \ll X H_{i}^{-1 / 3}+\log X
$$

Going back to (5.4) and (5.3), we finally obtain

$$
\mathcal{I}^{*}(X) \ll \sum_{i=0}^{l}\left(X H_{i}^{\varepsilon-1 / 3}+H_{i}^{\varepsilon} \log X\right) \ll X
$$

and

$$
\mathcal{I}(X) \ll X^{2}
$$

which completes the proof of Theorem 2 . 


\section{REFERENCES}

1. E. Bombieri and H. Iwaniec, On the order of $\zeta\left(\frac{1}{2}+i t\right)$, Ann. Scuola Norm. Sup. Pisa, Ser. IV, 13 (1986), 449-472.

2. M. Branton and P. Sargos, Points entiers au voisinage d'une courbe plane à très faible courbure, Bull. Sci. Math. 118 (1994), 15-28.

3. K. Chandrasekharan and R. Narasimhan, On the mean value of the error term for a class of arithmetic functions, Acta Math. 112 (1964), 41-67.

4. J. G. VAN DER CORPUT, Zahlentheoretische Abschätzungen mit Anwendung auf Gitterpunktprobleme, Math. Z. 17 (1923), 250-259. $215-254$.

5. J. G. VAN DER CORPUT, Neue zahlentheoretische Abschätzungen, Math. Ann. 89 (1923),

6. S. W. Graham and G. Kolesnik, Van der Corput's method of exponential sums (Cambridge University Press, Cambridge, 1991).

7. J. L. HAFNER, New omega theorems for two classical lattice point problems, Invent. Math. 63 (1981), 181-186.

8. J. L. HAFNER, On the average order of a class of arithmetical functions, J. Number Theory 15 (1982), 36-76.

9. E. HlawkA, Über die Zetafunktion konvexer Körper, Monatsh Math. 54 (1950), 100-107.

10. M. N. HuXLeY, Exponential sums and rounding error, J. London Math. Soc. (2) 43 (1991), 367-384.

11. M. N. Huxley, Exponential sums and lattice points II, Proc. London Math. Soc. 66 (1993), 279-301.

12. M. N. HuXley, The mean lattice point discrepancy, Proc. Edinburgh Math. Soc. 38 (1995), 523-531.

13. M. N. HuXLEY, Area, lattice points, and exponential sums (LMS Monographs, New Ser. 13, Oxford 1996).

14. M. N. HuXley and P. Sargos, Points entiers au voisinage d'une courbe plane de classe C $C^{n}$, Acta Arithm. 69 (1995), 359-366.

15. H. IWANIEC and C. J. MozzoChI, On the divisor and circle problems, J. Number Theory 29 (1988), 60-93.

16. I. KÁtaI, The number of lattice points in a circle, Ann. Univ. Sci. Budap. Rolando Eötvos, Sect. Math. 8 (1965), 39-60.

17. E. KräTzel, Lattice Points (Dt. Verl. d. Wiss., Berlin, 1988).

18. L. Kuipers and H. NiederReiter, Uniform distribution of sequences (J. Wiley \& Sons, New York 1974).

19. W. Müller, On the average order of the lattice rest of a convex body, Acta Arithm. 80 (1997), 89-100.

20. W. G. Nowak, Einige Beiträge zur Theorie der Gitterpunkte, in Zahlentheoretische Analysis (Seminar 1980-1982, ed. E. Hlawka, Springer LNM 1114, 1985), pp. 98-117.

21. W. G. Nowak, An $\Omega$-estimate for the lattice rest of a convex planar domain, Proc. Roy. Soc. Edinburgh Sect. A 100 (1985), 295-299. 
22. W. G. NowaK, On the average order of the lattice rest of a convex planar domain, Proc. Cambridge Philos. Soc. 98 (1985), 1-4.

23. D. Redmond, Mean value theorems for a class of Dirichlet series, Pacific J. Math. 78 (1978), 191-231.

24. H. P. F. SWINNERTON-DYeR, The number of lattice points on a convex curve, J. Number Theory 6 (1974), 128-135.

25. J. D. VaAler, Some extremal problems in Fourier analysis, Bull. Amer. Math. Soc. (2) 12 (1985), 183-216.

26. I. M. Vinogradov, Selected works (eds. L. D. Faddeev et al., Springer, BerlinHeidelberg 1985).

INSTITUT FÜR MATHEMATIK

UNIVERSITÄT FÜR BODENKULTUR

A-1180 WiEn, AUSTria

E-mail: nowak@mail.boku.ac.at 